\title{
Los trastornos mentales en América Latina y el Caribe: asunto prioritario para la salud pública
}

\author{
Robert Kohn, ${ }^{1}$ Itzhak Levav, ${ }^{2}$ José Miguel Caldas de Almeida, ${ }^{3}$ \\ Benjamín Vicente, ${ }^{4}$ Laura Andrade, ${ }^{5}$ Jorge J. Caraveo-Anduaga, ${ }^{6}$ \\ Shekhar Saxena ${ }^{7}$ y Benedetto Saraceno ${ }^{7}$
}

Forma de citar Kohn R, Levav I, Caldas de Almeida JM, Vicente B, Andrade L, Caraveo-Anduaga JJ, Saxena S, Saraceno B. Los trastornos mentales en América Latina y el Caribe: asunto prioritario para la salud pública. Rev Panam Salud Publica. 2005;18(4/5):229-40.

RESUMEN Objetivo. La creciente carga de trastornos mentales que afecta a las poblaciones de América Latina y el Caribe es demasiado grande para hacer caso omiso de ella. Por lo tanto, es una necesidad impostergable conocer la prevalencia de los trastornos mentales y la brecha de tratamiento, que está dada por la diferencia entre las tasas de prevalencia verdadera y las de las personas que han sido tratadas, que en algunos casos es grande pese a la existencia de tratamientos eficaces. Si se dispone de mayor informacion, se hace más factible 1) abogar mejor por los intereses de las personas que necesitan atención, 2) adoptar políticas más eficaces, 3) formular programas de intervención innovadores y 4) adjudicar recursos en conformidad con las necesidades observadas.

Métodos. Los datos se obtuvieron de estudios comunitarios publicados en América Latina y el Caribe entre 1980 y 2004. En esas investigaciones epidemiológicas se usaron instrumentos diagnósticos estructurados y se estimaron tasas de prevalencia. Las tasas brutas de diversos trastornos psiquiátricos en América Latina y el Caribe se estimaron a partir de las tasas media y mediana extraídas de los estudios, desglosadas por sexo. También se extrajeron los datos correspondientes al uso de servicios de salud mental para poder calcular la brecha en el tratamiento según trastornos específicos. Resultados. Las psicosis no afectivas (entre ellas la esquizofrenia) tuvieron una prevalencia media estimada durante el año precedente de 1,0\%; la depresión mayor, de 4,9\%; y el abuso o la dependencia del alcohol, de 5,7\%. Más de la tercera parte de las personas afectadas por psicosis no afectivas, más de la mitad de las afectadas por trastornos de ansiedad, y cerca de tres cuartas partes de las que abusaban o dependían del alcohol no habían recibido tratamiento psiquiátrico alguno, sea en un servicio especializado o en uno de tipo general.

Conclusiones. La actual brecha en el tratamiento de los trastornos mentales en América Latina y el Caribe sigue siendo abrumadora. Además, las tasas actuales probablemente subestiman el número de personas sin atención. La transición epidemiológica y los cambios en la composición poblacional acentuarán aun más la brecha en la atención en América Latina y el Caribe, a no ser que se formulen nuevas politicas de salud mental o que se actualicen las existentes, procurando incluir en ellas la extensión de los programas y servicios.

Palabras clave Salud mental, trastornos mentales, servicios de salud mental, recursos en salud, política de salud, América Latina, Región del Caribe.

1 Brown University, Department of Psychiatry and Human Behavior, Providence, Rhode Island, Estados Unidos de América.

2 Israel, Ministerio de Salud, Servicios de Salud Mental, Jerusalén, Israel.

3 Organización Panamericana de la Salud, División de Salud Mental y Promoción de la Salud, Washington, D.C., Estados Unidos de América.
4 Universidad de Concepción, Departamento de Psiquiatría, Concepción, Bío-Bío, Chile.

5 Departmento de Psiquiatría, Universidad de São Paulo, São Paulo, Brasil.

6 Instituto Mexicano de Psiquiatría, México, D.F., México.

7 Organización Mundial de la Salud, Departamento de Salud Mental y Abuso de Sustancias, Ginebra,
Suiza. La correspondencia debe mandarse a: Robert Kohn, Butler Hospital, 345 Blackstone Blvd., Providence, Rhode Island 02906, United States of America; teléfono: (401) 455-6277; fax: (401) 455-6566; correo electrónico: Robert_Kohn@ brown.edu 
La prevención, atención y rehabilitación de las personas afectadas por trastornos mentales constituyen un problema sanitario creciente en América Latina y el Caribe. Las bases epidemiológicas de una llamada a la acción se han hecho patentes durante el último decenio (1). En efecto, en 1990 se estimó que las afecciones psiquiátricas y neurológicas explicaban $8,8 \%$ de los años de vida ajustados por discapacidad (DALY) observados en América Latina y el Caribe (2). En 2002, esa carga había ascendido a más del doble, $22,2 \%$, según datos obtenidos del sitio web del proyecto Carga Mundial de Morbilidad de la Organización Mundial de la Salud (OMS). El excedente de discapacidad aportado por las enfermedades mentales obedece, en parte, a que aparecen a una edad más temprana que otras enfermedades crónicas (3). En América Latina y el Caribe esta carga, que es cada vez mayor, podría obedecer a la transición epidemiológica, es decir, al desplazamiento de las enfermedades transmisibles por las enfermedades crónicas; a que se presta mayor atención a ciertos trastornos emergentes, tales como la violencia y el sida; y a un cambio en la estructura poblacional que lleva a que un mayor número de personas alcancen la edad que las coloca en riesgo de sufrir los trastornos mentales propios de la adultez (4).

Con respecto a estos últimos cambios, cabe recordar que el Banco Mundial proyecta que entre 2000 y 2015 la población total de América Latina y el Caribe aumentará en 28\%, de 508 a 622 millones de personas (5). Esas proyecciones indican que el aumento poblacional más rápido se observará en el grupo de personas de edad más avanzada. La población de 50 años de edad o más aumentará en más de $60 \%$; la que se encuentra entre los 50 y 64 años de edad verá un aumento de $68 \%$, y la de 65 años o más ascenderá en $49 \%$. La población entre los 15 y 49 años de edad, que corre el mayor riesgo de sufrir los trastornos mentales que aparecen en la edad adulta, aumentará en $22 \%$.

Para poder hacerle frente a la creciente carga sanitaria que representan las enfermedades mentales en América Latina y el Caribe es menester entender su prevalencia y la brecha o hiato que existe en la provisión de atención, es decir, el porcentaje de personas que padecen de una afección determinada pero que no reciben ninguna atención. Algunos estudios epidemiológicos de tipo comunitario sobre los trastornos psiquiátricos que se han publicado desde los años ochenta arrojan luz sobre la magnitud de la carga. En ellos se calcularon las tasas de prevalencia de algunos trastornos mentales específicos en diversos países del continente americano mediante el uso de instrumentos de entrevista estructurados o semiestructurados ligados a los criterios diagnósticos actuales. Entre ellos figuran la Exploración del Estado Actual (Present State Examination, PSE) (6), el Instrumento de Entrevista Diagnóstica (Diagnostic Interview Schedule, DIS) (7) y la Entrevista Diagnóstica Internacional Compuesta (Composite International Diagnostic Interview, CIDI) (8). En el primer estudio de prevalencia efectuado en América Latina y el Caribe se utilizó el PSE (9-11). El advenimiento de criterios explícitos para el diagnóstico de las enfermedades mentales, tales como los pertenecientes a la Clasificación Internacional de Enfermedades (CIE) de la OMS (12) y al Manual Diagnóstico y Estadístico de los Trastornos Mentales de la Asociación Psiquiátrica Estadounidense (DSM-III, DSM-III-R y DSM-IV) (13-15), ha mejorado la fiabilidad y validez de los diagnósti-cos psiquiátricos. Los estudios epidemiológicos realizados en Brasil (16-20), Chile (21-27), Colombia (28-31), México (31-40), Perú (41-44) y Puerto Rico (45-47) establecieron tasas de prevalencia basadas en esos sistemas de diagnóstico. Dichos estudios se describen en el cuadro 1.

Los objetivos del presente informe son dos: 1) examinar las tasas de prevalencia basadas en estudios epidemiológicos comunitarios a fin de estimar las tasas agregadas para toda América Latina y el Caribe y (2) revisar los datos disponibles sobre el uso de los servicios de salud mental a fin de estimar la magnitud de la brecha en la atención psiquiátrica.

\section{MÉTODOS}

Para cumplir estos dos objetivos se escogieron los trastornos siguientes: las psicosis no afectivas, incluida la esquizofrenia; la depresión mayor; la distimia; el trastorno bipolar; el trastorno obsesivo-compulsivo; los trastornos de ansiedad generalizada; el abuso o la dependencia del alcohol; y el abuso o la dependencia de las drogas. Sobre la base de la información epidemiológica de los estudios en que se emplearon el PSE, el DIS y el CIDI, que se tradujeron al castellano y portugués, se estimaron el porcentaje y número de adultos de 15 años de edad o mayores que se ven afectados por trastornos psiquiátricos específicos en América Latina y el Caribe, sin contar la presencia de morbilidad múltiple, es decir, la presencia simultánea de dos o más trastornos en una misma persona. Se excluyeron los estudios basados en métodos de identificación y diagnóstico de casos que no se basaron en entrevistas a domicilio y en que no se utilizaron instrumentos diagnósticos estructurados o semiestructurados.

Los datos se extrajeron de revistas con arbitraje por pares, publicaciones gubernamentales, manuscritos en prensa y bases de datos del Consorcio Internacional de Epidemiología Psiquiátrica (CIEP). Se efectuaron búsquedas en MEDLINE y LILACS (ambas son bases de datos bibliográficas especializadas) para encontrar otros informes en inglés, portugués y español. También se buscaron otros materiales en la lista de referencias al final de cada una de las publicaciones revisadas.

Debido a sus implicaciones sanitarias complementarias, se examinaron las tasas de prevalencia correspondientes a tres períodos: 1) la tasa de prevalencia actual, correspondiente a la observada solo en el mes precedente; 2) la tasa de prevalencia correspondiente al año precedente, habiéndose incluido también trabajos que notificaban la prevalencia en los seis meses precedentes; y 3) la tasa de prevalencia de vida. Todos estos períodos de prevalencia tienen en común que representan la proporción de personas que podrían tener un determinado 


\section{CUADRO 1. Estudios epidemiológicos realizados en América Latina y el Caribe sobre la prevalencia de trastornos psiquiátricos}

\begin{tabular}{|c|c|c|c|c|c|c|}
\hline Estudio & $\begin{array}{c}\text { Referencia } \\
\text { bibliográfica }\end{array}$ & Fecha & $\begin{array}{c}\text { Tamaño y } \\
\text { características } \\
\text { de la muestra }\end{array}$ & $\begin{array}{l}\text { Edad } \\
\text { (años) }\end{array}$ & Instrumento & $\begin{array}{l}\text { Referencia } \\
\text { diagnóstica }\end{array}$ \\
\hline Bambuí, Brasil & 16 & 1996-1997 & $1041^{b}$ & $18+$ & CIDI & DSM-III-R \\
\hline Multicéntrico Brasil & 17,18 & 1991 & $6476^{c}$ & $15+$ & QMPA/DSM & DSM-III \\
\hline São Paulo, Brasil (CIDI) & 19,20 & 1994-1996 & $1464^{d}$ & $18+$ & CIDI 1.1 & DSM-III-R \\
\hline Santiago, Chile & 21 & 1996-1998 & $3870^{e}$ & $16-64$ & CIS-R & ClE-10 \\
\hline Colombia, depresión & 29 & 2000-2001 & $6610^{\mathrm{h}}$ & $18+$ & CIDI 2.1 & CIE-10 \\
\hline Colombia (WMH) & 30,31 & 2003 & $4544^{i}$ & $18-65$ & CIDI-CAPI & DSM-IV \\
\hline México (ENEP) & 31,32 & 2001-2002 & $5826^{j}$ & $18-65$ & CIDI-CAPI & DSM-IV \\
\hline México (PSE) & 33 & 1988 & $1984^{k}$ & $18-64$ & PSE & CIE-9 \\
\hline México, DF, México & $34-39$ & 1995 & $1937^{1}$ & $18-64$ & CIDI 1.1 & DSM-III-R \\
\hline México rural & 40 & 1996-1997 & $945^{\mathrm{m}}$ & $15-89$ & CIDI 1.1 & ClE-10 \\
\hline
\end{tabular}

a Buenos Aires, Argentina, características de la muestra: muestra de hogares estratificada por distritos. Esta fue una serie de encuestas comunitarias pequeñas con estratificación por distritos en los alrededores de Buenos Aires. Se encontró una prevalencia de punto sumamente alta de esquizofrenia de 3,0\%; de psicosis afectivas, de 4,0\%; de paranoia, de 0,2\%; de depresión neurótica, de 3,5\%; y de neurosis, de 14,5\%.

b Bambuí, Brasil, características de la muestra: muestra probabilística de hogares que abarcó a todos los habitantes de una población en el estado de Minas Gerais. Solamente se contempló la depresión, omitiendo el trastorno bipolar.

c Estudio multicéntrico en Brasil, características de la muestra: estudios individuales en tres grandes zonas urbanas: Brasilia, São Paulo y Porto Alegre. Se aplicó un diseño transversal bietápico. La primera etapa consistió en una entrevista de tamizaje con el Questionário de Morbidade Psiquiátrica do Adulto (QMPA) [Cuestionario de Morbilidad Psiquiátrica en Adultos], al que respondió cada miembro de familia mayor de 14 años. En la segunda etapa se llevó a cabo una entrevista diagnóstica estructurada elaborada por el quipo brasileño que se basó en el DSMIII y que se llevó a cabo en $30 \%$ de las personas que tuvieron resultados positivos y en $10 \%$ de las que tuvieron resultados negativos en la entrevista de tamizaje.

d São Paulo, Brasil, CIDI, características de la muestra: muestra probabilística estratificada de una zona de captación de los estratos socioeconómicos medio y alto, del Centro Médico de la Universidad de São Paulo. Hubo un sobremuestreo de personas de 18-24 y mayores de 59 años. Los autores proporcionaron más datos en cuadros posteriores.

e Santiago, Chile, características de la muestra: muestra probabilística de la población de Santiago. El instrumento usado fue la versión revisada del instrumento de entrevista clínica psiquiátrica semiestructurada (Clinical Interview Schedule-Revised, CIS-R).

f Chile, características de la muestra: muestreo polietápico de hogares efectuado en Chile en cuatro zonas de captación que son representativas de las principales zonas geográficas del país. Los autores proporcionaron más datos en cuadros posteriores.

g Colombia, características de la muestra: muestreo multietápico de hogares de todo el país.

h Colombia, estudio de depresión, características de la muestra: muestreo polietápico de hogares de todo el país. El estudio se centró en la prevalencia de la depresión.

Colombia, Salud Mental Mundial (World Mental Health, WMH), características de la muestra: muestreo polietápico de hogares de zonas urbanas en todo el país (Encuesta Mundial de Salud Mental). El instrumento usado fue el CIDI Computer-Assisted Personal Interview [Encuesta CIDI por Computadora] (CIDI-CAPI).

j México, Encuesta Nacional de Epidemiología Psiquiátrica ENEP (National Survey of Psychiatric Epidemiology), características de la muestra: muestreo polietápico de hogares de zonas urbanas en todo el país (Encuesta Mundial de Salud Mental).

k México, PSE, características de la muestra: muestreo de zonas urbanas con el instrumento de encuesta PSE/CATEGO y algunas preguntas tomadas de otras encuestas.

' México, DF, México, características de la muestra: muestreo polietápico de hogares en 16 divisiones políticas de la ciudad de México.

m México rural, características de la muestra: muestreo polietápico estratificado de 33 comunidades en dos estados de México.

n Lima, Perú, características de la muestra: muestreo polietápico del distrito de Independencia, que se ubica en el norte de Lima y que está habitado por personas de escasos recursos.

- Puerto Rico (Estados Unidos), características de la muestra: muestreo polietápico de hogares en Puerto Rico. En algunos de los informes se incluyó en la muestra a personas de 17 años.

trastorno y necesitar atención. De manera que, si bien la prevalencia actual subestima la proporción de personas que están en riesgo y necesitan atención, sirve, sin embargo, para identificar la frecuencia con que se presentan en las personas episodios agudos de un trastorno. Esta subestimación podría obedecer a la exclusión de personas que están asintomáticas en el momento de la entrevista o cuya afección ha dejado de satisfacer todos los criterios diagnósticos, quizá porque la persona ya ha recibido tratamiento.

2) La prevalencia correspondiente al año precedente podría constituir una aproximación a la proporción de per- sonas que necesitan atención. 3) En cuanto a la prevalencia de vida, esta podría subestimar la proporción de personas que han tenido trastornos, como resultado del sesgo de recordación (48). La utilidad de la prevalencia de vida depende de cuán crónico es un padecimiento determinado. Naturalmente, no toda persona en quien se diagnóstico un trastorno en algún momento de la vida requiere atención, ya que con el correr del tiempo el padecimiento podría haber desaparecido o menguado hasta un nivel subclínico.

En resumen, la prevalencia de vida proporciona información acerca de las personas que podrían necesitar trata- miento en cualquier momento durante la vida; la prevalencia correspondiente al año precedente proporciona información acerca de las necesidades de atención de la población en general; y la prevalencia en el momento actual (mes precedente) revela el número que necesita atención por un trastorno agudo. La mayor parte de los estudios no abarcan los tres tipos de prevalencia, y, como resultado, los datos usados para estimar las tasas de prevalencia para toda América Latina y el Caribe carecen de uniformidad.

En tres lugares (São Paulo, Brasil; México, D.F., México; y Chile) donde se realizaron estudios en los que se 
aplicó el CIDI como parte del CIEP, los investigadores han aportado datos que permiten calcular las tasas de prevalencia de los trastornos incluidos (38). Mediante un análisis de series de Taylor con el paquete estadístico SUDAAN (49), las tasas arrojadas por los estudios en estos tres lugares se ajustaron en función de diseños complejos y ponderaciones muestrales. Las tasas correspondientes a las psicosis no afectivas, a la esquizofrenia y a los trastornos relacionados con el abuso de sustancias en São Paulo (19) y México, D.F. (34) se obtuvieron de datos publicados, como también las tasas del trastorno obsesivo-compulsivo en México, D.F. (39). En el caso de los otros estudios, las tasas se basaron en informes publicados.

Las tasas de prevalencia varían ampliamente entre los distintos estudios debido a diferencias metodológicas y a posibles diferencias en los riesgos presentes en las poblaciones estudiadas. Por consiguiente, se usaron las tasas mediana y media de los diversos estudios a fin de representar la tasa aproximada en cada período de prevalencia. Esto dio por resultado un cálculo bruto, sin efectuarse ningún intento por ponderar los estudios en función de su valor metodológico. Se usó un solo informe representativo de cada estudio a fin de eliminar informes basados en muestras parciales.
Varios estudios publicaron tasas de prevalencia en distintas localidades o zonas de un mismo país; siempre que fue posible, se agruparon datos tomados de diferentes fuentes. En los demás casos se trató cada estudio por separado. Las estimaciones del número de personas afectadas no se ajustaron en función de la edad. Las tasas de América Latina y el Caribe se determinaron a partir de las tasas media y mediana de todos los estudios, desglosadas por sexo. Este uso doble se justifica en tanto que la tasa mediana permite eliminar los valores extremos de todos los estudios, mientras que la tasa media se basa en la premisa de que todos los estudios tienen la misma representatividad. Las tasas medianas constituyeron la base para calcular el número de personas afectadas por un determinado trastorno.

Los datos censales de cada país, distribuidos por edad y sexo, se obtuvieron del Anuario Demográfico de las Naciones Unidas (50). Se usó el cálculo censal más reciente provisto por cada país, habiéndose encontrado diferencias de un país a otro en cuanto al año del último censo. Se utilizaron las estimaciones preparadas por la OMS para el año 1999 al estimar las poblaciones actuales (51). Estas estimaciones solamente proporcionaron datos sobre la población total de cada país. La proporción de personas de 15 años o ma- yores de cada sexo obtenida de los datos censales se aplicó a las estimaciones de 1999 de la OMS, dando por sentado que esas proporciones no habían variado desde el último censo disponible.

Las estimaciones de la brecha de atención observada en América Latina y el Caribe se basaron en estudios que contenían datos sobre la utilización de servicios en función de diagnósticos específicos. Esa brecha está dada por la diferencia entre la tasa de prevalencia verdadera (personas con trastornos tratados y no tratados, según estudios comunitarios) y la tasa de personas con trastornos tratados.

\section{RESULTADOS}

\section{La carga de las enfermedades mentales}

Las psicosis no afectivas y la esquizofrenia se observaron en 1,4\% (media) a 1,6\% (mediana) de la población (cuadro 2) en algún momento de la vida. Las prevalencias correspondientes al último año fueron de $1,0 \%$ (media) y $7,0 \%$ (mediana). No se hallaron diferencias importantes en función del sexo.

La depresión mayor mostró una prevalencia media de $8,7 \%, 4,9 \%$ y $4,3 \%$ en algún momento de la vida, en

CUADRO 2. Tasas de prevalencia (\%) de psicosis no afectivas, según encuestas comunitarias en América Latina y el Caribe

\begin{tabular}{|c|c|c|c|c|c|c|c|c|c|}
\hline \multirow[b]{3}{*}{ Estudio } & \multicolumn{9}{|c|}{ Prevalencia } \\
\hline & \multicolumn{3}{|c|}{ En toda la vida } & \multicolumn{3}{|c|}{ Año precedente } & \multicolumn{3}{|c|}{ Actual } \\
\hline & Total & Hombres & Mujeres & Total & Hombres & Mujeres & Total & Hombres & Mujeres \\
\hline Buenos Aires, Argentina & $\ldots{ }^{a}$ & $\ldots$ & $\ldots$ & $\ldots$ & $\ldots$ & $\ldots$ & 4,0 & 3,0 & 4,3 \\
\hline Brasilia, Brasil & 0,3 & 0,0 & 0,5 & 0,2 & 0,0 & 0,4 & $\ldots$ & $\ldots$ & $\ldots$ \\
\hline Porto Alegre, Brasil & 2,4 & 2,4 & 2,5 & 2,0 & 2,4 & 2,0 & $\ldots$ & $\ldots$ & $\ldots$ \\
\hline São Paulo, Brasil & 0,9 & 0,0 & 1,2 & 0,6 & 0,0 & 1,2 & $\ldots$ & $\ldots$ & $\ldots$ \\
\hline São Paulo, Brasil (CIDI) & 1,9 & 1,7 & 2,0 & 0,8 & 0,8 & 0,9 & 0,7 & 0,5 & 0,8 \\
\hline Chile & 1,8 & 1,6 & 1,8 & 1,1 & 0,8 & 1,3 & 0,6 & 0,4 & 0,8 \\
\hline Colombia & 1,4 & 1,3 & 1,4 & 0,6 & 0,5 & 0,6 & $\ldots$ & $\ldots$ & $\ldots$ \\
\hline México (PSE) & $\ldots$ & $\ldots$ & $\ldots$ & $\ldots$ & $\ldots$ & $\ldots$ & 0,7 & 0,7 & 0,7 \\
\hline Lima, Perú & 0,6 & 0,3 & 1,0 & $0,6^{b}$ & $0,2^{b}$ & $1,0^{b}$ & $\ldots$ & $\ldots$ & $\ldots$ \\
\hline Puerto Rico & 1,8 & 2,2 & 1,4 & $1,7^{b}$ & $2,1^{b}$ & $1,3^{b}$ & $\ldots$ & $\ldots$ & $\ldots$ \\
\hline Media & 1,4 & 1,2 & 1,5 & 1,0 & 0,9 & 1,1 & 1,5 & 1,2 & 1,7 \\
\hline Mediana & 1,6 & 1,5 & 1,4 & 0,7 & 0,7 & 1,1 & 0,7 & 0,6 & 0,8 \\
\hline
\end{tabular}

a Las elipsis (. . . indican que no hubo observaciones.

b Tasa de prevalencia correspondiente a los 6 meses precedentes. 
CUADRO 3. Tasas de prevalencia (\%) de la depresión mayor, según encuestas comunitarias en América Latina y el Caribe

\begin{tabular}{|c|c|c|c|c|c|c|c|c|c|}
\hline \multirow[b]{3}{*}{ Study } & \multicolumn{9}{|c|}{ Prevalencia } \\
\hline & \multicolumn{3}{|c|}{ Toda la vida } & \multicolumn{3}{|c|}{ Año precedente } & \multicolumn{3}{|c|}{ Actual } \\
\hline & Total & Hombres & Mujeres & Total & Hombres & Mujeres & Total & Hombres & Mujeres \\
\hline Buenos Aires, Argentina & $\ldots^{a}$ & $\ldots$ & $\ldots$ & $\ldots$ & $\ldots$ & $\ldots$ & 6,0 & 6,0 & 6,0 \\
\hline Bambuí, Brasil & 12,8 & 7,3 & 17,0 & 9,1 & 5,1 & 12,2 & 7,5 & 5,1 & 10,2 \\
\hline Brasilia, Brasil & 2,8 & 1,9 & 3,8 & 1,5 & 1,1 & 2,9 & $\ldots$ & $\ldots$ & $\ldots$ \\
\hline Porto Alegre, Brasil & 10,2 & 5,9 & 14,5 & 1,3 & 0,0 & 3,6 & $\ldots$ & $\ldots$ & $\ldots$ \\
\hline São Paulo, Brasil & 1,9 & 0,0 & 3,8 & 6,7 & 5,9 & 7,6 & $\ldots$ & $\ldots$ & $\ldots$ \\
\hline São Paulo, Brasil (CIDI) & 12,6 & 8,8 & 15,3 & 5,8 & 3,6 & 7,5 & 3,9 & 2,7 & 4,8 \\
\hline Santiago, Chile & $\ldots$ & $\ldots$ & $\ldots$ & $\ldots$ & $\ldots$ & $\ldots$ & 5,5 & 2,7 & 8,0 \\
\hline Chile & 9,2 & 6,8 & 11,5 & 5,7 & 3,7 & 7,5 & 3,4 & 2,3 & 4,5 \\
\hline Colombia & 19,6 & 18,3 & 20,7 & 1,9 & 0,7 & 3,0 & $\ldots$ & $\ldots$ & $\ldots$ \\
\hline Colombia, depresión & $\ldots$ & $\ldots$ & $\ldots$ & 10,0 & 6,2 & 12,0 & 8,5 & 5,3 & 10,2 \\
\hline Colombia (WMH) & 12,1 & 8,6 & 14,9 & 5,6 & 3,5 & 7,3 & 1,9 & 0,7 & 2,8 \\
\hline México (PSE) & $\ldots$ & $\ldots$ & $\ldots$ & $\ldots$ & $\ldots$ & $\ldots$ & 3,2 & 2,2 & 3,9 \\
\hline México (ENEP) & 3,3 & 2,0 & 4,5 & 1,4 & 0,9 & 2,1 & 0,6 & 0,3 & 0,8 \\
\hline México, DF, México & 8,1 & 5,5 & 10,1 & 4,4 & 3,1 & 5,6 & 2,2 & 1,4 & 2,8 \\
\hline México rural & 6,2 & 2,9 & 9,1 & $\cdots$ & $\ldots$ & $\ldots$ & $\ldots$ & $\ldots$ & $\ldots$ \\
\hline Lima, Perú & 9,7 & 6,1 & 13,5 & $5,2^{b}$ & $3,4^{b}$ & $6,9^{b}$ & $\ldots$ & $\ldots$ & $\ldots$ \\
\hline Puerto Rico & 4,6 & 3,5 & 5,5 & $2,3^{b}$ & $2,4^{b}$ & $3,3^{b}$ & $\ldots$ & $\ldots$ & $\ldots$ \\
\hline Media & 8,7 & 6,0 & 11,1 & 4,9 & 3,1 & 6,5 & 4,3 & 2,9 & 5,4 \\
\hline Mediana & 9,2 & 5,9 & 11,5 & 5,4 & 3,5 & 7,1 & 3,7 & 2,4 & 4,7 \\
\hline
\end{tabular}

a Las elipsis (. . .) indican que no hubo observaciones.

${ }^{\mathrm{b}}$ Tasa de prevalencia correspondiente a los 6 meses precedentes.

el año precedente $\mathrm{y}$ en el momento actual (mes precedente), respectivamente. Las tasas medianas observadas en estos períodos de prevalencia fueron de $9,2 \%, 5,4 \%$ y $3,7 \%$, respectivamente (cuadro 3). La frecuencia de la enfermedad fue casi el doble en mujeres. La tasa de prevalencia de distimia fue la mitad de la observada en el caso de la depresión mayor (cuadro 4). En el caso del trastorno bipolar, la tasa de prevalencia de vida tuvo una media de $1,4 \%$ y la tasa de prevalencia en el año precedente tuvo una media de $0,8 \%$ (cuadro 5). Las tasas medianas fueron casi idénticas. No se detectaron diferencias en función del sexo en la prevalencia media o mediana de tras- torno bipolar, aunque hubo grandes diferencias entre un estudio y otro.

Los trastornos de ansiedad generalizada mostraron una alta prevalencia en la población y fueron más frecuentes en mujeres que en varones. Las tasas medias de prevalencia a lo largo de la vida y durante el último año, estimadas en $5,5 \%$ y $3,4 \%$, fueron el doble

CUADRO 4. Tasas de prevalencia (\%) de distimia, según encuestas comunitarias en América Latina y el Caribe

\begin{tabular}{|c|c|c|c|c|c|c|c|c|c|}
\hline \multirow[b]{3}{*}{ Estudio } & \multicolumn{9}{|c|}{ Prevalencia } \\
\hline & \multicolumn{3}{|c|}{ Toda la vida } & \multicolumn{3}{|c|}{ Año precedente } & \multicolumn{3}{|c|}{ Actual } \\
\hline & Total & Hombres & Mujeres & Total & Hombres & Mujeres & Total & Hombres & Mujeres \\
\hline São Paulo, Brasil (CIDI) & 4,9 & 4,2 & 5,5 & 1,9 & 1,8 & 2,1 & 1,6 & 1,6 & 1,5 \\
\hline Chile & 8,0 & 3,5 & 12,1 & 3,9 & 1,6 & 5,9 & 2,9 & 1,5 & 4,2 \\
\hline Colombia (WMH) & 0,7 & 0,6 & 0,7 & 0,5 & 0,5 & 0,5 & 0,1 & 0,1 & 0,2 \\
\hline México (ENEP) & 0,9 & 0,5 & 1,1 & 0,4 & 0,4 & 0,5 & 0,2 & 0,2 & 0.2 \\
\hline México, DF, México & 1,4 & 0,9 & 1,8 & 0,3 & 0,3 & 0,4 & 0,2 & 0,0 & 0,3 \\
\hline México rural & 3,4 & 1,4 & 5,2 & $\ldots a$ & $\ldots$ & $\ldots$ & $\ldots$ & $\ldots$ & $\ldots$ \\
\hline Lima, Perú & 3,6 & 1,0 & 5,9 & $3,3^{b}$ & $1,0^{b}$ & $5,9^{b}$ & $\ldots$ & $\ldots$ & $\ldots$ \\
\hline Puerto Rico & 4,7 & 1,6 & 7,6 & $\ldots$ & $\ldots$ & $\ldots$ & $\ldots$ & $\ldots$ & $\ldots$ \\
\hline Media & 3,5 & 1,7 & 5,0 & 1,7 & 0,9 & 2,6 & 1,0 & 0,7 & 1,3 \\
\hline Mediana & 3,5 & 1,2 & 5,4 & 1,2 & 0,8 & 1,3 & 0,2 & 0,2 & 0,3 \\
\hline
\end{tabular}

\footnotetext{
a Las elipsis (. . .) indican que no hubo observaciones.

${ }^{b}$ Tasa de prevalencia correspondiente a los 6 meses precedentes.
} 
CUADRO 5. Tasas de prevalencia (\%) del trastorno bipolar, según encuestas comunitarias en América Latina y el Caribe

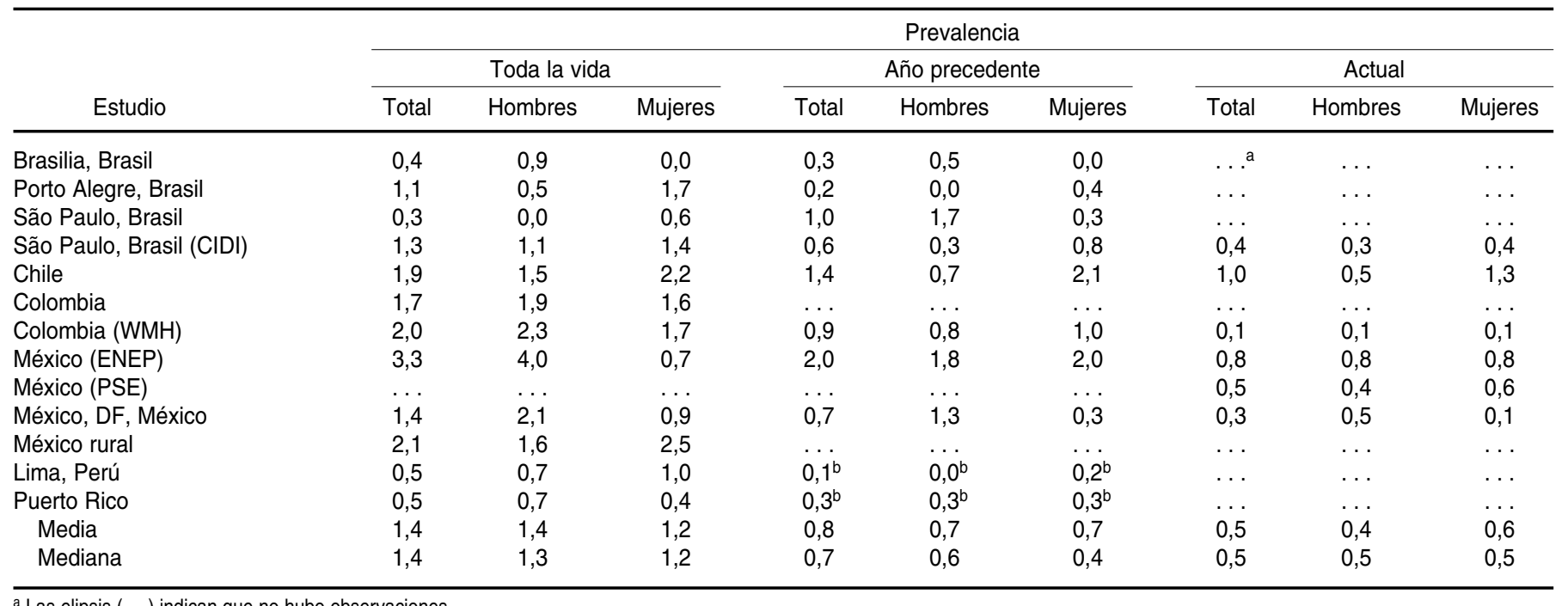

${ }^{a}$ Las elipsis (. . .) indican que no hubo observaciones.

${ }^{\mathrm{b}}$ Tasa de prevalencia correspondiente a los 6 meses precedentes.

CUADRO 6. Tasas de prevalencia (\%) de los trastornos de ansiedad generalizada, según encuestas comunitarias en América Latina y el Caribe

\begin{tabular}{|c|c|c|c|c|c|c|c|c|c|}
\hline \multirow[b]{3}{*}{ Estudio } & \multicolumn{9}{|c|}{ Prevalencia } \\
\hline & \multicolumn{3}{|c|}{ Toda la vida } & \multicolumn{3}{|c|}{ Año precedente } & \multicolumn{3}{|c|}{ Actual } \\
\hline & Total & Hombres & Mujeres & Total & Hombres & Mujeres & Total & Hombres & Mujeres \\
\hline Porto Alegre, Brasil & 9,6 & 5,2 & 14,0 & 5,4 & 5,2 & 5,6 & $\ldots$ & $\ldots$ & $\ldots$ \\
\hline São Paulo, Brasil & 10,6 & 7,3 & 13,9 & 6,9 & 4,3 & 9,6 & & $\ldots$ & $\ldots$ \\
\hline São Paulo, Brasil (CIDI) & 2,5 & 1,8 & 3,0 & 1,0 & 0,7 & 1,3 & 0,9 & 0,7 & 1,1 \\
\hline Colombia & 3,1 & 2,6 & 3,5 & 1,3 & 1,7 & 0,9 & & $\ldots$ & $\ldots$ \\
\hline Colombia (WMH) & 1,3 & 1,5 & 1,2 & 0,7 & 0,9 & 0,5 & 0,3 & 0,5 & 0,2 \\
\hline México (ENEP) & 1,2 & 0,7 & 1,6 & 0,7 & 0,6 & 0,8 & 0,4 & 0,6 & 0,3 \\
\hline México, DF, México & 0,9 & 0,8 & 1,0 & 0,6 & 0,7 & 0,4 & 0,2 & 0,0 & 0,3 \\
\hline Media & 5,5 & 3,8 & 7,1 & 3,4 & 2,5 & 4,2 & 1,3 & 0,9 & 1,7 \\
\hline Mediana & 2,6 & 1,8 & 3,5 & 1,3 & 0,9 & 1,3 & 0,7 & 0,6 & 0,7 \\
\hline
\end{tabular}

${ }^{a}$ Las elipsis (...) indican que no hubo observaciones.

de las tasas medianas, $2,6 \%$ y $1,3 \%$, respectivamente (cuadro 6). Las crisis de pánico mostraron una tasa de prevalencia de vida de $1,6 \%$ y una tasa de prevalencia durante el año precedente de 1,0\% (cuadro 7). Este trastorno afecta más frecuentemente a personas de sexo femenino. En cambio, no se detectaron diferencias en la tasa de prevalencia del trastorno obsesivocompulsivo en función del sexo. Este trastorno tuvo tasas de prevalencia de vida de $1,9 \%$ (media) y de $1,8 \%$ (mediana); las tasas de prevalencia durante el año precedente fueron de 1,4\% (media) y de 1,2\% (mediana) (cuadro 8).

La tasa media de prevalencia de vida del abuso o la dependencia del alcohol se estimó en 11,3\% y la prevalencia media durante el año precedente, en 5,7\%, observándose tasas de prevalencia mucho mayores en hombres que en mujeres (cuadro 9). De 1,5\% a 1,6\% de la población se vió afectada por trastornos relacionados con el abuso de drogas en algún momento de la vida; $0,5 \%$ a 0,7\% había tenido trastornos de ese tipo durante el año anterior. Se advirtieron tasas mucho más altas en hombres que en mujeres (cuadro 10).

A partir de 1999, la población total de adultos (de 15 años o mayores) en América Latina y el Caribe se estimó 
CUADRO 7. Tasas de prevalencia (\%) de los trastornos de pánico, según encuestas comunitarias en América Latina y el Caribe

\begin{tabular}{|c|c|c|c|c|c|c|c|c|c|}
\hline \multirow[b]{3}{*}{ Estudio } & \multicolumn{9}{|c|}{ Prevalencia } \\
\hline & \multicolumn{3}{|c|}{ Toda la vida } & \multicolumn{3}{|c|}{ Año precedente } & \multicolumn{3}{|c|}{ Actual } \\
\hline & Total & Hombres & Mujeres & Total & Hombres & Mujeres & Total & Hombres & Mujeres \\
\hline São Paulo, Brasil (CIDI) & 1,3 & 0,2 & 2,2 & 1,0 & 0,2 & 1,6 & 0,5 & 0,0 & 0,9 \\
\hline Santiago, Chile & $\ldots^{a}$ & $\ldots$ & $\ldots$ & $\ldots$ & $\ldots$ & $\ldots$ & 1,3 & 1,1 & 1,5 \\
\hline Chile & 1,6 & 0,7 & 2,5 & 0,9 & 0,5 & 1,2 & 0,6 & 0,3 & 0,8 \\
\hline Colombia & 0,3 & 0,3 & 0,2 & 0,1 & 0,2 & 0,1 & $\ldots$ & $\ldots$ & $\ldots$ \\
\hline Colombia (WMH) & 1,2 & 0,6 & 1,7 & 0,6 & 0,3 & 0,9 & 0,3 & 0,1 & 0,5 \\
\hline México (ENEP) & 2,1 & 1,3 & 2,9 & 1,1 & 0,4 & 1,7 & 0,4 & 0,2 & 0,5 \\
\hline México, DF, México & 2,9 & 2,1 & 3,8 & 1,6 & 0,8 & 1,9 & 0,4 & 0,1 & 0,7 \\
\hline Lima, Perú & 1,6 & 1,2 & 2,9 & $1,6^{b}$ & $1,0^{b}$ & $2,2^{b}$ & $\ldots$ & $\ldots$ & $\ldots$ \\
\hline Puerto Rico & 1,7 & 1,6 & 1,9 & $1,1^{\mathrm{b}}$ & $1,2^{b}$ & $0,9^{b}$ & $\ldots$ & $\ldots$ & $\ldots$ \\
\hline Media & 1,6 & 1,0 & 2,3 & 1,0 & 0,6 & 1,3 & 0,6 & 0,3 & 0,8 \\
\hline Mediana & 1,6 & 1,0 & 2,4 & 1,1 & 0,5 & 1,4 & 0,5 & 0,2 & 0,8 \\
\hline
\end{tabular}

a Las elipsis (. . .) indican que no hubo observaciones.

b Tasa de prevalencia correspondiente a los 6 meses precedentes.

CUADRO 8. Tasas de prevalencia (\%) del trastorno obsesivo-compulsivo, según encuestas comunitarias en América Latina y el Caribe

\begin{tabular}{|c|c|c|c|c|c|c|c|c|c|}
\hline \multirow[b]{3}{*}{ Estudio } & \multicolumn{9}{|c|}{ Prevalencia } \\
\hline & \multicolumn{3}{|c|}{ Toda la vida } & \multicolumn{3}{|c|}{ Año precedente } & \multicolumn{3}{|c|}{ Actual } \\
\hline & Total & Hombres & Mujeres & Total & Hombres & Mujeres & Total & Hombres & Mujeres \\
\hline Brasilia, Brasil & 0,9 & 0,7 & 0,5 & 0,5 & 0,5 & 0,4 & $\ldots{ }^{a}$ & $\ldots$ & $\ldots$ \\
\hline Porto Alegre, Brasil & 2,1 & 1,7 & 2,5 & 1,2 & 1,7 & 0,7 & $\ldots$ & $\ldots$ & $\ldots$ \\
\hline São Paulo, Brasil (CIDI) & 0,6 & 0,4 & 0,8 & 0,6 & 0,4 & 0,7 & 0,5 & 0,4 & 0,5 \\
\hline Santiago, Chile & $\ldots$ & $\ldots$ & $\ldots$ & $\ldots$ & $\ldots$ & $\ldots$ & 1,3 & 1,4 & 1,1 \\
\hline Chile & 1,2 & 0,7 & 1,6 & 1,2 & 0,7 & 1,6 & 1,2 & 0,7 & 1,6 \\
\hline Colombia & 3,6 & 3,3 & 3,9 & 3,1 & 2,4 & 3,8 & $\ldots$ & $\ldots$ & $\ldots$ \\
\hline México (PSE) & $\ldots$ & $\ldots$ & $\ldots$ & $\ldots$ & $\ldots$ & $\ldots$ & 3,0 & 1,3 & 4,3 \\
\hline México, DF, México & 1,4 & 0,8 & 1,7 & 1,0 & 0,7 & 1,2 & $\ldots$ & $\ldots$ & $\ldots$ \\
\hline Lima, Perú & 2,2 & 2,0 & 2,9 & $1,5^{b}$ & $1,5^{b}$ & $1,5^{b}$ & $\ldots$ & $\ldots$ & $\ldots$ \\
\hline Puerto Rico & 3,2 & 3,3 & 3,1 & $1,8^{b}$ & $1,3^{b}$ & $2,3^{b}$ & $\ldots$ & $\ldots$ & $\ldots$ \\
\hline Media & 1,9 & 1,6 & 2,1 & 1,4 & 1,2 & 1,5 & 1,5 & 1,0 & 1,9 \\
\hline Mediana & 1,8 & 1,3 & 2,1 & 1,2 & 1,0 & 1,4 & 1,3 & 1,0 & 1,4 \\
\hline
\end{tabular}

a Las elipsis (. . .) indican que no hubo observaciones.

b Tasa de prevalencia correspondiente a los 6 meses precedentes.

en 338 millones (165 millones de varones y 173 millones de mujeres). En el cuadro 11 se presentan las estimaciones del número de adultos afectados por cada trastorno, por sexo, en cada uno de los tres períodos de prevalencia examinados. Los trastornos que afectaron al mayor número de adultos fueron la depresión mayor, la distimia, los trastornos de ansiedad generalizada, y el abuso o la dependencia del alcohol. Por ejemplo, en algún momento de la vida, la depresión mayor y el abuso o la dependencia del alcohol afectaron individualmente a alrededor de 31,1 millones de adultos.

\section{La brecha de atención de los trastornos mentales}

En América Latina y el Caribe, la brecha de atención de los trastornos mentales es grande. Los estudios que aportaron datos sobre el déficit de tratamiento dieron cifras correspondientes a la utilización de servicios en distintos períodos, entre ellos el último mes, los últimos seis meses, el último año y toda la vida. En el cuadro 12 se presentan las respectivas brechas en la atención de ocho trastornos en diferentes lugares, así como la mediana y la media de la brecha encontrada en los distintos estudios. En las muestras examinadas, más de la tercera parte de las personas con una psicosis no afectiva, más de la mitad de las que padecían trastornos de ansiedad y alrededor de tres cuartas partes de las que tenían problemas relacionados con el consumo de alcohol no recibieron nin- 
CUADRO 9. Tasas de prevalencia (\%) del abuso o la dependencia del alcohol, según encuestas comunitarias en América Latina y el Caribe

\begin{tabular}{|c|c|c|c|c|c|c|c|c|c|}
\hline \multirow[b]{3}{*}{ Estudio } & \multicolumn{9}{|c|}{ Prevalencia } \\
\hline & \multicolumn{3}{|c|}{ Toda la vida } & \multicolumn{3}{|c|}{ Año precedente } & \multicolumn{3}{|c|}{ Actual } \\
\hline & Total & Hombres & Mujeres & Total & Hombres & Mujeres & Total & Hombres & Mujeres \\
\hline Porto Alegre, Brasil & 9,2 & 16,0 & 2,5 & 8,7 & 15,9 & 1,6 & $\ldots$ & $\ldots$ & $\ldots$ \\
\hline São Paulo, Brasil & 7,6 & 15,2 & 0,0 & 4,3 & 8,6 & 0,0 & $\ldots$ & $\ldots$ & $\ldots$ \\
\hline São Paulo, Brasil (CIDI) & 14,9 & 24,4 & 7,9 & 9,9 & 15,9 & 5,4 & 7,5 & 12,0 & 4,1 \\
\hline Colombia (WMH) & 9,0 & 17,9 & 1,9 & 2,2 & 4,6 & 0,4 & 0,7 & 1,6 & 0,1 \\
\hline México (ENEP) & 8,3 & 16,4 & 1,3 & 2,3 & 4,7 & 0,2 & 0,6 & 1,3 & 0,1 \\
\hline México, DF, México & 9,1 & 19,4 & 1,3 & 5,6 & 10,9 & 2,2 & 2,6 & 5,7 & 0,3 \\
\hline Lima, Perú & 18,7 & 34,8 & 2,5 & $6,9^{b}$ & $12,5^{b}$ & $1,2^{b}$ & $\cdots$ & $\cdots$ & $\ldots$ \\
\hline Puerto Rico & 12,6 & 24,6 & 2,0 & 6,1 & $\ldots$ & $\ldots$ & $\ldots$ & $\ldots$ & $\ldots$ \\
\hline Media & 11,3 & 20,6 & 2,9 & 5,7 & 9,8 & 1,9 & 3,4 & 5,9 & 1,3 \\
\hline Mediana & 9,2 & 17,9 & 2,0 & 5,6 & 9,8 & 1,4 & 2,6 & 5,7 & 0,3 \\
\hline
\end{tabular}

a Las elipsis (. . . indican que no hubo observaciones.

${ }^{b}$ Tasa de prevalencia correspondiente a los 6 meses precedentes.

CUADRO 10. Tasas de prevalencia (\%) del abuso o dependencia de drogas ilícitas, según encuestas comunitarias en América Latina y el Caribe

\begin{tabular}{|c|c|c|c|c|c|c|}
\hline \multirow[b]{3}{*}{ Estudio } & \multicolumn{6}{|c|}{ Prevalencia } \\
\hline & \multicolumn{3}{|c|}{ Toda la vida } & \multicolumn{3}{|c|}{ Año precedente } \\
\hline & Total & Hombres & Mujeres & Total & Hombres & Mujeres \\
\hline São Paulo, Brasil (CIDI) & $1, .0$ & 1,9 & 0,6 & 0,6 & 1,1 & 0,2 \\
\hline Chile & 3,5 & 3,4 & 3,5 & 1,8 & 1,7 & 2,1 \\
\hline Colombia (WMH) & 1,6 & 2,3 & 0,3 & 0,5 & 0,9 & 0,2 \\
\hline México (ENEP) & 1,5 & 3,1 & 0,2 & 0,5 & 1,0 & 0,0 \\
\hline México, DF, México & 0,7 & $\ldots{ }^{\mathrm{a}}$ & $\ldots$ & & & \\
\hline Lima, Perú & 1,5 & 2,2 & 0,7 & $0,2^{b}$ & $0,2^{b}$ & $0,2^{b}$ \\
\hline Puerto Rico & 1,2 & 2,2 & 0,4 & $\ldots$ & $\ldots$ & $\ldots$ \\
\hline Media & 1,6 & 2,5 & 1,0 & 0,7 & 1,0 & 0,5 \\
\hline Mediana & 1,5 & 2,3 & 0,5 & 0,5 & 1,0 & 0,2 \\
\hline
\end{tabular}

b Tasa de prevalencia correspondiente a los 6 meses precedentes.

gún tratamiento psiquiátrico en el sistema de salud formal.

\section{DISCUSIÓN}

Este informe sufre de varias limitaciones. Hay gran variedad en la calidad y metodología de los estudios incluidos en él. Además, se aplicaron los datos de un número reducido de países a fin de representar las tasas de prevalencia y el número de personas afectadas en toda América Latina y el Caribe. Asimismo, muchos de los estudios no fueron de alcance nacional, pero se dio por sentado que su población era representativa del país en su totalidad. A ello se suman las disparidades entre países y dentro de muchos de los países de América Latina y el Caribe en términos económicos, sociales y culturales, y en la organización, disponibilidad y cobertura de los servicios de salud. No obstante, se dio por sentado que estas diferencias no afectaban a la prevalencia de los trastornos mentales ni a la magnitud de la brecha de tratamiento. Por ejemplo, existe una relación inversa entre el estrato socioeco- nómico y la prevalencia de muchos trastornos psiquiátricos (52) y es posible que esa relación inversa también se refleje en la búsqueda de atención especializada. Además, otros trastornos que no se incluyeron en este ejercicio podrían constituir una carga no despreciable. Entre esos trastornos se encuentran las demencias en los adultos mayores y las enfermedades psiquiátricas en niños y adolescentes, con sus respectivas consecuencias para quienes cuidan a las personas afectadas.

A pesar de que en los distintos estudios se examinó la búsqueda de trata- 
CUADRO 11. Número de adultos (de 15 años de edad o más) (en millones) en América Latina y el Caribe que tienen enfermedades mentales, calculado a partir de las tasas medianas de los estudios examinados

\begin{tabular}{|c|c|c|c|c|c|c|c|c|c|}
\hline \multirow[b]{3}{*}{ Enfermedad } & \multicolumn{9}{|c|}{ Prevalencia } \\
\hline & \multicolumn{3}{|c|}{ Toda la vida } & \multicolumn{3}{|c|}{ Año precedente } & \multicolumn{3}{|c|}{ Actual } \\
\hline & Total & Hombres & Mujeres & Total & Hombres & Mujeres & Total & Hombres & Mujeres \\
\hline Depresión mayor & 31,1 & 9,7 & 19,9 & 18,3 & 5,8 & 12,3 & 12,5 & 4,0 & 8,1 \\
\hline Distimia & 11,8 & 2,0 & 9,3 & 4,1 & 1,3 & 2,2 & 0,7 & 0,3 & 0,5 \\
\hline Trastorno bipolar & 4,7 & 2,1 & 2,1 & 2,4 & 1,0 & 0,7 & 1,7 & 0,8 & 0,9 \\
\hline Trastorno obsesivo-compulsivo & 6,1 & 2,1 & 3,6 & 4,1 & 1,7 & 2,4 & 4,4 & 1,7 & 2,4 \\
\hline Abuso o dependencia del alcohol & 31,1 & 29,6 & 3,5 & 18,9 & 16,2 & 2,4 & 8,8 & 9,4 & 0,5 \\
\hline Abuso o dependencia de drogas & 5,1 & 3,8 & 0,9 & 1,7 & 1,7 & 0,3 & $\ldots^{\mathrm{a}}$ & $\ldots$ & $\ldots$ \\
\hline
\end{tabular}

a Las elipsis (. . .) indican que no hubo observaciones.

CUADRO 12. Brecha en el tratamiento de las enfermedades mentales en América Latina y el Caribe, expresada en términos del porcentaje de personas que necesitaban tratamiento y no lo recibieron ${ }^{\mathrm{a}}$

\begin{tabular}{|c|c|c|c|c|c|c|c|c|}
\hline Enfermedad & $\begin{array}{l}\text { São Paulo } \\
\text { (mes } \\
\text { precedente) }\end{array}$ & $\begin{array}{c}\text { Chile } \\
\text { (6 meses } \\
\text { precedentes) }\end{array}$ & $\begin{array}{c}\text { México } \\
\text { ENEP } \\
\text { (año } \\
\text { precedente) }\end{array}$ & $\begin{array}{c}\text { México, } \\
\text { DF } \\
\text { (toda } \\
\text { la vida) }\end{array}$ & $\begin{array}{l}\text { México } \\
\text { rural } \\
\text { (toda } \\
\text { la vida) }\end{array}$ & $\begin{array}{l}\text { Puerto Rico } \\
\qquad \text { (año } \\
\text { precedente) }\end{array}$ & Media & Mediana \\
\hline Psicosis no afectivas & 58,0 & 44,4 & $\ldots b$ & $\ldots$ & $\ldots$ & 9,7 & 37,4 & 44,4 \\
\hline Depresión mayor & 49,4 & 46,2 & 78,2 & 43,4 & 66,3 & 70,0 & 58,9 & 57,9 \\
\hline Distimia & 43,8 & 32,4 & 81,5 & 78,5 & 58,0 & $\ldots$ & 58,8 & 58.0 \\
\hline Trastorno bipolar & 46,0 & 50,2 & 85,7 & 74,1 & $\ldots$ & $\ldots$ & 64,0 & 62,2 \\
\hline Ansiedad generalizada & 41,1 & 44,2 & 94,7 & 72,2 & $\ldots$ & $\ldots$ & 63,1 & 58,2 \\
\hline $\begin{array}{l}\text { Trastornos de pánico } \\
\text { Trastorno }\end{array}$ & 47,8 & 22,7 & 71,2 & 70,0 & $\ldots$ & $\cdots$ & 52,9 & 58,9 \\
\hline $\begin{array}{l}\text { obsesivo-compulsivo } \\
\text { Abuso o dependencia }\end{array}$ & $\ldots$ & 27,6 & $\ldots$ & 92,1 & $\ldots$ & $\cdots$ & 59,9 & 59,9 \\
\hline del alcohol & 53,3 & 84,8 & $\ldots$ & $\ldots$ & $\ldots$ & 76,0 & 71,4 & 76,0 \\
\hline
\end{tabular}

a La encuesta WMH, realizada en Colombia, publicó datos que revelan la brecha en el tratamiento (durante el año previo a la encuesta) de las siguientes afecciones exclusivamente: cualquier tipo de trastorno afectivo, 82,4\%; cualquier tipo de trastorno de ansiedad, 95,4\%; y cualquier tipo de abuso de sustancias, $92,5 \%$.

${ }^{b}$ Las elipsis (. . . ) indican que no hubo observaciones.

miento en diversos períodos, la brecha observada en la atención es sumamente grande. En el caso de afecciones particulares esa brecha podría estar subestimada, ya que no se tuvo en cuenta la posible coexistencia de más de una afección en una misma persona. Seguramente, la brecha identificada sería aun mayor si los estudios se hubieren realizado en el nivel nacional y no solo local y si la información hubiese provenido de todos los países y no solamente de unos pocos que poseen mayores recursos, incluso para la investigación epidemiológica. Por ejemplo, un estudio en Belice en que se examinó la prevalencia del tratamiento y que se basó en una revisión de los archivos médicos de todos los proveedores de servicios de salud que trataban a pacientes con enfermedades mentales reveló que cerca de $63 \%$ de las personas con esquizofrenia no recibían tratamiento alguno. Lo mismo se aplicaba a $89 \%$ de las que padecían trastornos afectivos y a $99 \%$ de las que tenían trastornos de ansiedad (53).

Más datos que confirman la insuficiencia de los servicios de salud mental en América Latina y el Caribe fueron arrojados por el Proyecto Atlas, dirigido por la OMS $(54,55)$. En mu- chos países de América Latina y el Caribe, el gasto en la salud mental y el número de camas para pacientes psiquiátricos, de psiquiatras y de otros profesionales de la salud mental son mucho menores que en Canadá y Estados Unidos (cuadro 13).

A juzgar por nuestros resultados, la situación de la salud mental en América Latina y el Caribe constituye un reto de grandes proporciones para la salud pública que se acentúa a medida que va cambiando la estructura poblacional. De hecho, la planificación racional en el ámbito de la salud mental implica tener en cuenta las necesi- 
CUADRO 13. Recursos invertidos en la atención de la salud mental en países de América Latina y el Caribe, por comparación con Canadá y Estados Unidos

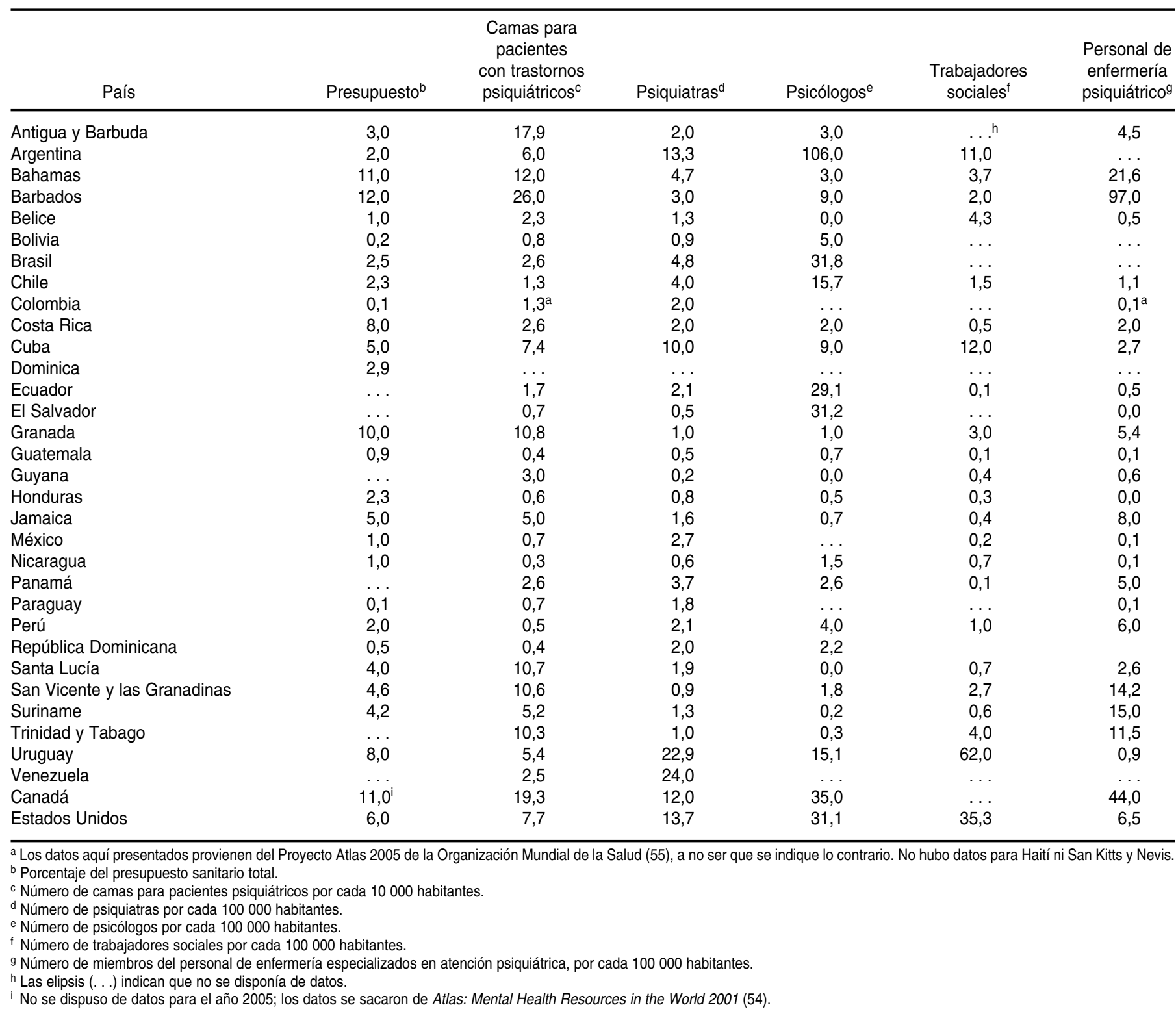

dades futuras a la luz de la situación actual.

La carga de enfermedades mentales de la población no es solamente un reflejo de la prevalencia de estados psicopatológicos, sino el resultado de un conjunto de estados y problemas en los cuales el comportamiento desempeña un papel preponderante. El sector de la salud mental siempre se ha dedicado exclusivamente a los trastornos psiquiátricos y a los que caen bajo la categoría más amplia de los trastornos neuropsiquiátricos, tales como la epilepsia. Algunos países, dependiendo de sus recursos y de su forma habitual de llevar a cabo la planificación, limitan lo programas de salud mental al tratamiento exclusivo de los trastornos mentales más graves. Una visión tan estrecha de lo que constituye la salud mental podría ser insuficiente hoy en día. Del campo de la salud mental se espera que desempeñe un papel cada vez más importante en la prevención y el tratamiento de las enfermedades crónicas y emergentes en las cuales hay un componente conductual.

En los países industrializados se observa una clara tendencia a darle mayor relevancia a la atención de la salud mental y se está haciendo hincapié en la detección de los problemas en los servicios de atención primaria, en la creación de programas de medicina conductual y en la salud de las perso- 
nas de edad avanzada. El papel que desempeña el comportamiento en la salud en general no tiene menos importancia en países en vías de industrializarse que en otros lugares, pero pasa desapercibido como resultado de la excesiva dependencia de un modelo médico estrecho y de la escasez de especialistas en las ciencias de la conducta.

A medida que aumenta el número de personas con trastornos mentales y que sufren cambios, al menos parciales, los factores de riesgo, es posible que el uso de servicios también se modifique. Por otro lado, la demanda de servicios ya está cambiando a medida que los países abogan por dar de alta a personas recluidas en hospitales psiquiátricos por tener enfermedades mentales crónicas. Es posible que la demanda de servicios aumente a raíz del movimiento a favor de una defini- ción comunitaria de la responsabilidad por el cuidado de la salud mental, unida a una mejor aceptación social de las enfermedades mentales y de las personas que las padecen. La incipiente alianza en pro de la salud mental, dentro y fuera del sector sanitario, que la OMS recomienda continuar y fortalecer (56) permitirá hacer frente a las necesidades actuales y futuras de una manera más eficaz.

\section{REFERENCIAS}

1. Levav I, Lima BR, Somoza Lennon M, Kramer M, González R. Salud mental para todos en América Latina y el Caribe. Bases epidemiológicas para la acción. Bol Oficina Sanit Panam. 1989;107:196-219.

2. Murray CJL, Lopez AD, eds. The global burden of disease: a comprehensive assessment of mortality and disability from diseases, injuries, and risk factors in 1990 and projected to 2020. Cambridge, MA: Escuela de Salud Pública de la Universidad de Harvard en nombre de la Organización Mundial de la Salud y el Banco Mundial; 1996.

3. WHO International Consortium in Psychiatric Epidemiology. Cross-national comparisons of the prevalences and correlates of mental disorders. Bull World Health Organ. 2000;78: 413-25.

4. Pan American Health Organization. Health in the Americas 2002. Washington, D.C.: PAHO; 2002.

5. World Bank. World development indicators 2002. New York: World Bank; 2002.

6. Wing JH, Nixon J, Mann SA, Leff JP. Reliability of the PSE (ninth edition) used in a population survey. Psychol Med. 1977;7:505-16.

7. Robins LN, Helzer JE, Croughan J, Ratcliff KS. National Institute of Mental Health Diagnostic Interview Schedule: its history, characteristics, and validity. Arch Gen Psychiatry. 1981; 38:381-9.

8. Robins LN, Wing J, Wittchen HU, Helzer JE, Babor TF, Burke J, et al. The Composite International Diagnostic Interview: an epidemiologic instrument suitable for use in conjunction with different diagnostic systems and in different cultures. Arch Gen Psychiatry. 1988; 45:1069-77.

9. Larraya FP, Casullo MM, Viola FP. Prevalencia de la patología mental en la megalópolis de Buenos Aires. Buenos Aires: Consejo Nacional de Investigaciones Científicas y Técnicas; 1982.

10. Di Marco G. Prevalencia de desórdenes mentales en el área metropolitana de la República Argentina. Acta Psiquiatr Psicol Am Lat. 1982; 28:93-102.

11. Aszkenazi M, Casullo MM. Factores socioculturales y presencia de psicopatologías en poblaciones de distintas localidades argentinas. Acta Psiquiatr Psicol Am Lat. 1984;30:11-20.
12. World Health Organization. The ICD-10 classification of mental and behavioural disorders: clinical descriptions and diagnostic guidelines. Geneva: WHO; 1992.

13. American Psychiatric Association. Diagnostic and statistical manual of mental disorders. $3 .^{\mathrm{a}}$ ed. Washington, D.C.: APA; 1980.

14. American Psychiatric Association. Diagnostic and statistical manual of mental disorders. 3 . $^{\mathrm{a}}$ ed., revisada. Washington, D.C.: APA; 1987.

15. American Psychiatric Association. Diagnostic and statistical manual of mental disorders. $4 .^{\text {a }}$ ed. Washington, D.C.: APA; 1994.

16. Vorcaro CM, Lima-Costa MF, Barreto SM, Uchoa E. Unexpected high prevalence of 1-month depression in a small Brazilian community: the Bambuí Study. Acta Psychiatr Scand. 2001;104:257-63.

17. Almeida Filho N, Mari JJ, Coutinho E, França JF, Fernandes JG, Andreoli SB, et al. Estudo multicêntrico de morbidade psiquiátrica em áreas urbanas brasileiras (Brasília, São Paulo, Porto Alegre). Rev ABPAPAL. 1992;14: 93-104.

18. Almeida-Filho N, Mari J de J, Coutinho E, Franca JF, Fernandes J, Andreoli SB, et al. Brazilian multicentric study of psychiatric morbidity. Br J Psychiatry. 1997;171:524-9.

19. Andrade LH, Lolio CA, Gentil V, Laurenti R. Epidemiologia dos transtornos mentais em uma área definida de captação da cidade de São Paulo, Brasil. Rev Psiquiatr Clin. 1999;26: 257-62.

20. Andrade L, Walters EE, Gentil V, Laurenti R. Prevalence of ICD-10 mental disorders in a catchment area in the city of São Paulo, Brazil. Soc Psychiatry Psychiatr Epidemiol. 2002;37: 316-25.

21. Araya R, Rojas G, Fritsch R, Acuña J, Lewis G. Common mental disorders in Santiago, Chile: prevalence and socio-demographic correlates. Br J Psychiatry. 2001;178:228-33.

22. Rioseco P, Escobar B, Vicente B, Vielma M, Saldivia S, Cruzat M, et al. Prevalencia de vida de algunos trastornos psiquiátricos en la provincia de Santiago. Rev Psiquiatr. 1994;11: $186-93$.

23. Vicente B, Rioseco P, Vielma M, Uribe M, Boggiano $G$, Torres $S$. Prevalencia de vida de algunos trastornos psiquiátricos en la provincia de Concepción. Rev Psiquiatr. 1992;9:1050-60.
24. Saldivia S, Vicente B, Kohn R, Rioseco P, Torres $\mathrm{S}$. Use of mental health services in Chile. Psychiatr Serv. 2004;55:71-6.

25. Vicente B, Kohn R, Rioseco P, Saldivia S, Baker C, Torres S. Population prevalence of psychiatric disorders in Chile: 6-month and 1-month rates. Br J Psychiatry. 2004;184: 299-305.

26. Vicente B, Rioseco P, Saldivia S, Kohn R, Torres S. Estudio chileno de prevalencia de patología psiquiátrica (DSM-III-R/CIDI) (ECPP). Rev Med Chil. 2002;130:527-36.

27. Bjil RV, de Graff R, Hiripi E, Kessler RC, Kohn $\mathrm{R}$, Offord DR, et al. The prevalence of treated and untreated mental disorders in five countries. Health Aff (Millwood). 2003;22:122-33.

28. Torres de Galvis Y, Montoya ID. Segundo Estudio Nacional de Salud Mental y Consumo de Sustancias Psicoactivas, Colombia, 1997. Santa Fe de Bogotá: Ministerio de Salud; 1997.

29. Gómez-Restrepo C, Bohóquez A, Pinto Masis D, Gil Laverde JFA, Rondón Sepúlveda M, Díaz-Granados N. Prevalencia de depresión y factores asociados con ella en la población colombiana. Rev Panam Salud Publica. 2004;16: 378-86.

30. Posada-Villa JA, Aguilar-Gaxiola SA, Magaña CG, Gómez LC. Prevalencia de trastornos mentales y uso de servicios: resultados preliminares del Estudio nacional de salud mental Colombia 2003. Rev Colomb Psiquiatr. 2004; 33:241-62.

31. Demyttenaere K, Bruffaerts R, Posada-Villa J, Gasquet I, Kovess V, Lepine JP, et al. Prevalence, severity, and unmet need for treatment of mental disorders in the World Health Organization World Mental Health Surveys. JAMA. 2004;291:2581-90.

32. Medina-Mora ME, Borges G, Muñoz CL, Benjet $C$, Jaimes JB, Bautista CF, et al. Prevalencia de trastornos mentales y uso de servicios: resultados de la encuesta nacional de epidemiología psiquiátrica en México. Salud Ment. 2003;26:1-16

33. Caraveo-Anduaga J, Medina-Mora ME, Rascón ML, Villatoro J, Martínez-Vélez A, Gómez M. La prevalencia de los trastornos psiquiátricos en la población urbana adulta en México. Salud Ment. 1996;19(3):14-21.

34. Caraveo-Anduaga JJ. Epidemiología de la morbilidad psiquiátrica en la Ciudad de Mé- 
xico. México: Instituto Mexicano de Psiquiatría; 1995.

35. Caraveo-Anduaga JJ, Martínez Vélez NA, Rivera Guevara BE, Dayan AP. Prevalencia en la vida de episodios depresivos y utilización de servicios especializados. Salud Ment. 1997;20 (Supl 2):15-23.

36. Caraveo-Anduaga JJ, Colmenares E, Saldívar GJ. Morbilidad psiquiátrica en la ciudad de México: prevalencia y comorbilidad a lo largo de la vida. Salud Ment. 1999;22(número especial):62-7.

37. Merikangas KR, Mehta RL, Mohar BE, Walters EE, Swendsen JD, Aguilar-Gaxiola, et al. Comorbidity of substance use disorders with mood and anxiety disorders: results of the International Consortium in Psychiatric Epidemiology. Addict Behav. 1998;23:893-907.

38. Andrade L, Caraveo-Anduaga JJ, Berglund P, Bijl RV, De Graaf R, Vollebergh W, et al. The epidemiology of major depressive episodes: results from the International Consortium of Psychiatric Epidemiology (ICPE) Surveys. Int J Methods Psychiatr Res. 2003;12:3-21.

39. Caraveo-Anduaga JJ, Bermúndez EC. The epidemiology of obsessive-compulsive disorder in Mexico City. Salud Ment. 2004;27(2):1-6.

40. Salgado de Snyder VN, Díaz-Pérez M. Los trastornos afectivos en la población rural. Salud Ment. 1999;22(número especial):68-74.

41. Hayashi S, Perales A, Sogi C, Warthon D, Llanos R, Novara J. Prevalencia de vida de tras- tornos mentales en Independencia (Lima, Perú). An Salud Ment. 1985;1:206-22.

42. Minobe K, Perales A, Sogi C, Warthon D, Llanos R, Sato T. Prevalencia de vida de trastornos mentales en Independencia (Lima, Perú). An Salud Ment. 1990;6:9-20.

43. Yamamoto J, Silva JA, Sasao T, Wang C, Nguyen L. Alcoholism in Peru. Am J Psychiatry. 1993;150:1059-62.

44. Perales A, Sogi C. Epidemiología psiquiátrica en el Perú. An Salud Ment. 1995;11:9-29.

45. Canino GJ, Bird HR, Shrout PE, Rubio-Stipec M, Bravo M, Martinez R, et al. The prevalence of specific psychiatric disorders in Puerto Rico. Arch Gen Psychiatry. 1987;44:727-35.

46. Canino G, Anthony JC, Freeman Dh, Shrout P, Rubio-Stipec M. Drug abuse and illicit drug use in Puerto Rico. Am J Public Health. 1993; 83:194-200.

47. Alegría M, Kessler RC, Bijl R, Lin E, Heeringa SG, Takeuchi DT, et al. Comparing data on mental health service use between countries. In: Andrews G, Henderson S, eds. Unmet need in psychiatry. New York: Cambridge University Press; 2000. Pp. 97-118.

48. Andrews G, Anstey K, Brodaty H, Issakidis C, Luscombe G. Recall of depressive episode 25 years previously. Psychol Med. 1999;29: 787-91.

49. Shah BV, Barnwell BG, Bieler GS. SUDAAN user's manual. Release 7.5. Research Triangle Park: Research Triangle Institute; 1997.
50. United Nations. Demographic yearbook historical supplement 1948-1997. New York: $\mathrm{UN} ; 2000$.

51. World Health Organization. The world health report 2000-health systems: improving performance. Geneva: WHO; 2000.

52. Kohn R, Dohrenwend BP, Mirotznik J. Epidemiologic findings on selected psychiatric disorders in the general population. En: Dohrenwend BP, ed. Adversity, stress, and psychopathology. New York: Oxford University Press; 1998 . Pp. 235-84.

53. Bonander J, Kohn R, Arana B, Levav I. An anthropological and epidemiological overview of mental health in Belize. Transcult Psychiatry. 2000;37:57-72.

54. World Health Organization. Atlas: mental health resources in the world 2001. Geneva: WHO; 2001.

55. World Health Organization. Mental health atlas-2005. Geneva: WHO; 2005.

56. World Health Organization. The world health report 2001-mental health: new understanding, new hope. Geneva: WHO; 2001.

Manuscrito recibido el 22 de marzo de 2004 y aceptado para publicación, tras revisión, el 22 de abril de 2005.
ABSTRACT

Mental disorders in Latin America and the Caribbean: a public health priority

Key words
Objective. The growing burden of mental disorders in Latin America and the Caribbean has become too large to ignore. There is a need to know more about the prevalence of mental disorders and the gap between the number of individuals with psychiatric disorders and the number of those persons who remain untreated even though effective treatments exist. Having that knowledge would make it possible to improve advocacy, adopt better policies, formulate innovative intervention programs, and apportion resources commensurate with needs.

Methods. Data were extracted from community-based psychiatric epidemiological studies published in Latin America and the Caribbean from 1980 through 2004 that used structured diagnostic instruments and provided prevalence rates. Estimates of the crude rates in Latin America and the Caribbean for the various disorders were determined by calculating the mean and median rates across the studies, by gender. In addition, data on service utilization were reviewed in order to calculate the treatment gap for specific disorders.

Results. Nonaffective psychosis (including schizophrenia) had an estimated mean one-year prevalence rate of $1.0 \%$; major depression, $4.9 \%$; and alcohol use abuse or dependence, $5.7 \%$. Over one-third of individuals with nonaffective psychosis, over half of those with an anxiety disorder, and some three-fourths of those with alcohol use abuse or dependence did not receive mental health care from either specialized or general health services.

Conclusions. The current treatment gap in mental health care in Latin America and the Caribbean remains wide. Further, current data likely greatly underestimate the number of untreated individuals. The epidemiological transition and changes in the population structure will further widen the treatment gap in Latin America and the Caribbean unless mental health policies are formulated or updated and programs and services are expanded.

Mental health, mental disorders, mental health services, health resources, health policy, Latin America, Caribbean Region. 